\title{
Diaphragm Electrical Activity During Expiration in Mechanically Ventilated Infants
}

\author{
GUILLAUME EMERIAUD, JENNIFER BECK, MARISA TUCCI, JACQUES LACROIX, AND CHRISTER SINDERBY
}

\begin{abstract}
Department of Pediatrics [G.E., MT, J.L.], Hôpital Sainte-Justine, Quebec, Canada, H3T 1C5; Department of Pediatrics [G.E.], Centre Hospitalier Universitaire, Grenoble 38043, France; Department of Newborn and Developmental Pediatrics [J.B.], Sunnybrook and Women's College Health Sciences Centre, Toronto, Ontario, Canada M5S 1B2; Hôpital Sainte-Justine Research Center [M.T., J.L.], Université de Montréal, Montreal, Quebec, Canada, H3T 1C5; Department of Critical Care Medicine [C.S.], St-Michael’s Hospital, University of Toronto, Toronto, Ontario, Canada M5B 1 W8
\end{abstract}

\begin{abstract}
The presence of diaphragm electrical activity (EAdi) during expiration is believed to be involved in the maintenance of end-expiratory lung volume (EELV) and has never been studied in intubated and mechanically ventilated infants. The aim of this study was to quantify the amplitude of diaphragm electrical activity present during expiration in mechanically ventilated infants and to measure the impact of removing positive end-expiratory pressure (PEEP) on this activity. We studied the EAdi in 16 ready-to-be weaned intubated infants who were breathing on their prescribed ventilator and PEEP settings. In all 16 patients, 5 min of data were collected on the prescribed ventilator settings. In a subset of eight patients, the PEEP was briefly reduced to zero PEEP (ZEEP). EAdi was recorded with miniaturized sensors placed on a conventional nasogastric feeding tube. Airway pressure (Paw) was also measured. For each spontaneous breath, we identified the neural inspiration and neural expiration. Neural expiration was divided into quartiles (Q1, Q2, Q3, and Q4), and the amplitude of EAdi calculated for each Q1-Q4 represented $95 \pm 29 \%, 31 \pm 15 \%, 15 \pm 8 \%$, and $12 \pm 7 \%$, respectively, of the inspiratory EAdi amplitude. EAdi for Q3-Q4 significantly increased during ZEEP, and decreased after reapplication of PEEP. These findings confirm that the diaphragm remains partially active during expiration in intubated and mechanically ventilated infants and that removal of PEEP affects this tonic activity. This could have potential implications on the management of PEEP in intubated infants. (Pediatr Res 59: 705-710, 2006)
\end{abstract}

$\mathrm{T}$ he diaphragm is primarily known as an inspiratory muscle that is active during inspiration and relaxed during expiration. However, in healthy premature and full-term newborns, it has been demonstrated that electrical activity of the diaphragm, measured with electrodes on the surface of the chest wall, may persist throughout expiration, suggesting a "tonic" activity of the respiratory muscles (1-4). Lopes et al. (2) demonstrated that changes in tonic activity of the diaphragm were associated with changes in EELV and provided the original physiologic explanation that persistent diaphragmatic activity during the exhalation period helps to regulate EELV in infants . In healthy full-term or premature infants,

Received August 12, 2005; accepted December 23, 2005.

Correspondence: Jennifer Beck, M.D., 40 Humberview Road, Toronto, Ontario, Canada M6S 1W6; e-mail: Jennifer.beck@rogers.com.

Supported by the Canadian Intensive Care Foundation, and Fondation de l'Hôpital Sainte-Justine. the EELV is higher than the relaxation volume, the latter being determined by the passive mechanical properties of the respiratory system $(1,3,5)$. It is only after $1 \mathrm{y}$ of age that the EELV coincides with the relaxed lung volume (6); before this age, adapting reflexes must compensate for the reduced EELV. In newborns, persistence of diaphragm activity during expiration, in combination with the flow-braking action of the laryngeal adductor muscles (7), a high respiratory rate, and a reduced time-constant of the respiratory system, has been suggested to contribute to an active elevation of EELV (5,811). However, in intubated and mechanically ventilated infants, the presence of an endotracheal tube does not allow for expiratory flow braking via the laryngeal muscles and likely increases their dependence on tonic diaphragm activity (i.e. diaphragm activity during exhalation). To our knowledge, no data on this have yet been reported in human infants.

The aims of the present study were to (1) develop a method to quantify the EAdi during expiration in mechanically ventilated infants (i.e. tonic diaphragm activity) and (2) determine whether tonic EAdi is present during prescribed PEEP and whether removal of PEEP produces changes in tonic EAdi.

\section{METHODS}

Subjects. Sixteen patients (11 males, five females) with various diagnoses were included (Table 1). Patients were intubated and mechanically ventilated in synchronized intermittent mandatory ventilation (SIMV) mode with a Draeger Babylog 8000 ventilator (Draeger, Lubeck, Germany). The patient inclusion criteria were infants recovering from acute respiratory failure deemed ready for extubation, aged $3 \mathrm{~d}-6$ mo. According to Colin et al. (6), the Hering-Breuer reflexes are present and strong for this age range. The patients were breathing with the same ventilator parameters for at least $2 \mathrm{~h}$ before the study. Patients with neuromuscular disease, bleeding disorders, cardiovascular instability, depressed central respiratory drive, esophageal malformations, and phrenic nerve damage as well as patients having undergone esophageal surgery were excluded.

The protocol was approved by the ethical and scientific committees of Sainte-Justine Hospital, Montreal, Quebec, Canada. Written informed consent was obtained from the parents of all patients.

Measurements. Electrical activity of the crural diaphragm (EAdi) was obtained using nine miniaturized electrodes mounted on a conventional

Abbreviations: EAdi, electrical activity of the diaphragm; EELV, endexpiratory lung volume; Paw, airway pressure; PEEP, positive end-expiratory pressure; SIMV, synchronized intermittent mandatory ventilation; Te, expiratory time; Ti, inspiratory time; ZEEP, zero PEEP

DOI: 10.1203/01.pdr.0000214986.82862.57 
Table 1. Patient anthropometrical data, ventilator settings, diagnosis, and tonic diaphragm activity

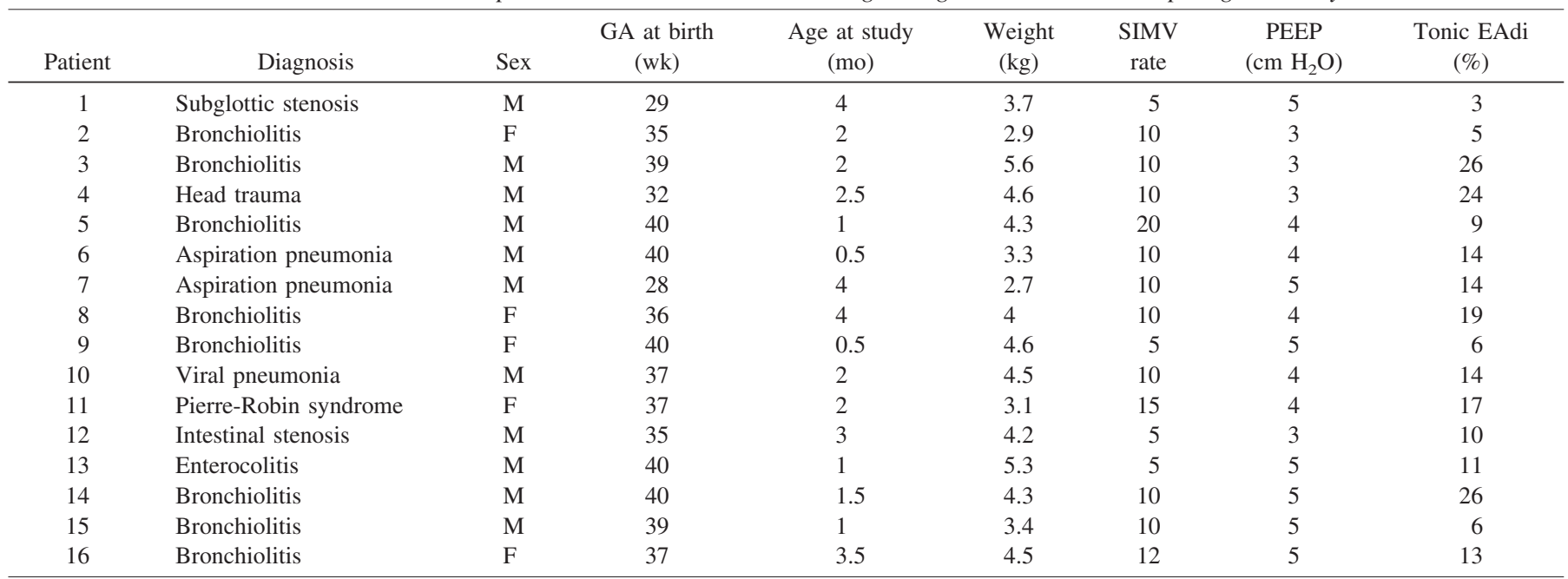

EAdi is expressed as a percentage of EAdi observed during the prepeak period,after subtraction of the noise level.

GA, gestational age at birth.

nasogastric (8 French) feeding tube (Benlan Inc., Oakville, Ontario, Canada), and spaced $5 \mathrm{~mm}$ apart. Paw was measured from a side port of the endotracheal tube (Sensym Inc., Milpitas, $\mathrm{CA} ; \pm 350 \mathrm{~cm} \mathrm{H}_{2} \mathrm{O}$ ). The EAdi and Paw signals were fed into a computer for data acquisition and online display.

Protocol. Studies were conducted a few hours before extubation, and patients had not received sedation for at least $6 \mathrm{~h}$ before the test. Following endotracheal aspiration, the feeding tube with the electrodes was inserted. Optimal electrode positioning was achieved by online feedback of the EAdi signals (12).

Following catheter insertion, the infants continued to breathe on the prescribed ventilator and PEEP settings. Ten minutes after catheter placement, recordings of EAdi and Paw were obtained in all 16 subjects during a 5-min period on the prescribed PEEP.

In eight patients, a 1-min period of ZEEP was introduced at the end of the 5 -min period to determine its influence on tonic EAdi. Following this period, the patients were returned to their prescribed PEEP level.

Data acquisition and signal analysis. EAdi and Paw data were acquired, processed online, and displayed. EAdi signals were continuously and automatically processed with algorithms described by Sinderby et al. (13-15). The EAdi signal strength was quantified by the root mean square. The "true" noise level of the signal was estimated during short periods of apnea, defined as periods where both EAdi and Paw signals were flat for $>1 \mathrm{~s}$ (Fig. 1).

For the conditions of prescribed PEEP and ZEEP, we analyzed the spontaneous breaths only and excluded the mandatory breaths and the postmandatory breaths. For each spontaneous breath, we identified and placed time cursors for (1) the onset of EAdi, (2) the peak of EAdi, and (3) the onset of the subsequent neural inspiratory effort, as previously described (16) (Fig. 1). We excluded all breaths where this identification was problematic due to

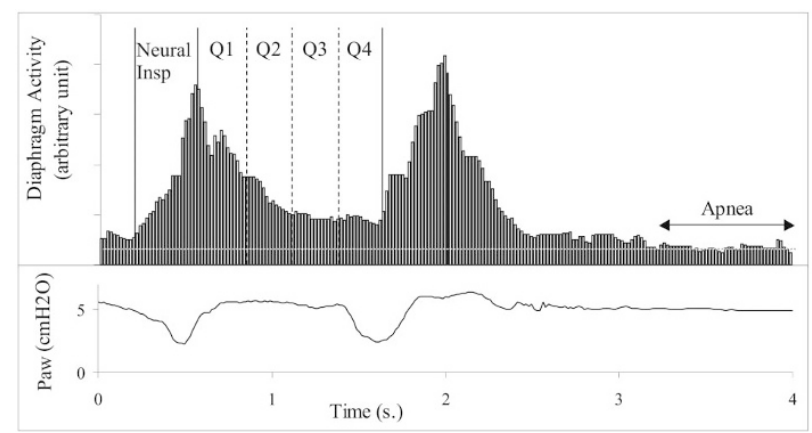

Figure 1. Definition of the periods of analysis for each spontaneous breath. Neural inspiration and expiration were defined by the diaphragm electrical activity (see text for details). Neural expiration was divided into four quartiles (Q1, Q2, Q3, and Q4). Vertical solid lines identify the onset and the peak of EAdi. Vertical dashed lines separate the quartiles. Horizontal dashed lines represent the noise level. Pao, airway opening pressure. interference with electrocardiographic signal. Neural inspiratory time (Ti) and neural expiratory time (Te) were defined from the EAdi tracings (Fig. 1). The entire neural Ti was used to calculate the mean inspiratory amplitude of EAdi. To quantify the EAdi amplitude during neural expiration, neural Te was divided into quartiles (Q1-Q4) (Fig. 1), and the mean EAdi amplitude was calculated for each quartile. The mean EAdi of each quartile was expressed in percentage of the mean inspiratory EAdi, after subtraction of the noise level.

Statistical analysis. Data were analyzed by two independent investigators (G.E. and a technician). The agreement between observers was estimated with the intraclass correlation coefficients (ICCs), calculated with Deyo's method (17) for each EAdi quartile. Interobserver reproducibility is considered to be good when ICC $>0.75$ (17).

In all 16 patients, the mean inspiratory EAdi, and the mean EAdi for Q1-Q4 were calculated for the $5 \mathrm{~min}$ of the prescribed PEEP period. In the subgroup of eight patients, during ZEEP, data were analyzed during the minute preceding ZEEP (PreZEEP), the minute during ZEEP (ZEEP), and the minute after ZEEP (PostZEEP). Statistical analysis was performed with commercially available software (Statview 5.0, SAS Institute Inc.). One-way repeated-measures analysis of variance (ANOVA) was used to determine differences between the different quartiles during prescribed PEEP and between PreZEEP, ZEEP, and PostZEEP. The difference between a quartile and the common noise level was estimated with a paired $t$ test. A $p$ value $<0.05$ was considered to be significant. All data are expressed as mean and SD $($ mean $\pm \mathrm{SD})$.

\section{RESULTS}

The patient data are summarized in Table 1 . Their mean ( $\pm \mathrm{SD}$ ) age, height, and weight were $2.3 \pm 1.3 \mathrm{mo}, 54 \pm 4$ $\mathrm{cm}$, and $4.0 \pm 0.8 \mathrm{~kg}$, respectively. The baseline ventilator settings are presented in Table 1. The mean prescribed $\mathrm{FiO}_{2}$ for the group was $35 \pm 13 \%$.

Determination of tonic EAdi at prescribed level of PEEP $(\boldsymbol{n}=\mathbf{1 6})$. The average SIMV rate was $10.0 \pm 4.3$ breaths per minute and the average prescribed PEEP level was $3.9 \pm 0.8$ $\mathrm{cm} \mathrm{H}_{2} \mathrm{O}$.

Figure 1 shows an example of EAdi and Paw tracings for two consecutive spontaneous breaths followed by an apnea period and depicts how diaphragm activation persists (above the noise level) throughout the neural expiratory period. The pattern of tonic EAdi could attain a relatively constant level as shown in Figure 1, but could also be very variable as depicted in Figure 2 for several patients.

The mean EAdi amplitude calculated during Q1, Q2, Q3, and Q4 is shown in Figure $3(n=16)$. The EAdi observed 

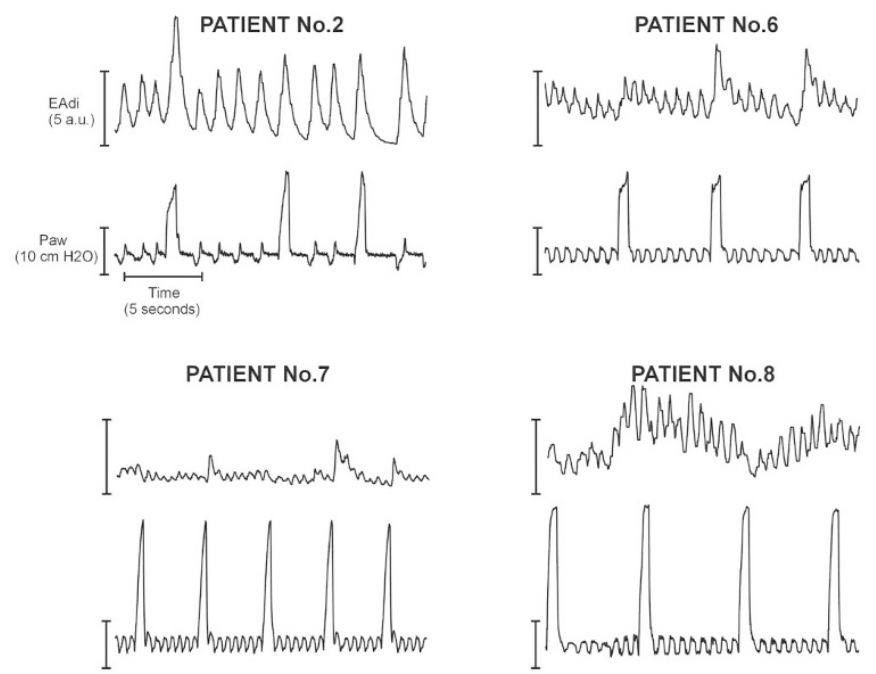

Figure 2. Tracings of diaphragm activity and Paw. Representative tracings of EAdi and Paw in four infants breathing on the prescribed ventilator settings.

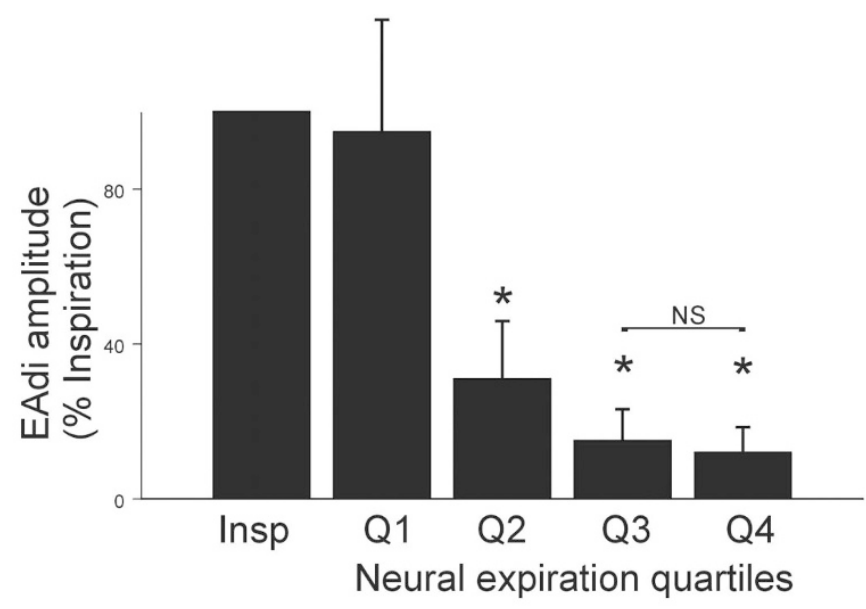

Figure 3. Group mean $( \pm \mathrm{SD})$ diaphragm electrical activity during inspiration and the expiratory quartiles $(n=16)$. Activity is expressed as a percentage of the mean inspiratory amplitude after subtraction of noise level. All quartiles showed significant magnitudes above noise level $(p<0.05)$. No difference was observed between inspiratory EAdi and Q1 or between Q3 and Q4. ${ }^{*} p<0.05$, significant from inspiration.

during Q1 was similar to the EAdi observed during the neural inspiratory period. EAdi decreased during Q2 $(p<0.05)$, to stabilize at its lowest level during Q3 and Q4 $(p<0.05)$. No differences were observed between $\mathrm{Q} 3$ and $\mathrm{Q} 4$, which constituted $15 \pm 8 \%$ and $12 \pm 7 \%$ of inspiratory EAdi, respectively. All quartiles showed significant magnitudes above the noise level $(p<0.05)$. The mean noise level for the group was $0.8 \pm 0.3$ arbitrary units (range, $0.3-1.4$ ).

Activity during Q1 was more correlated with the inspiratory EAdi than were the later quartiles; the correlation coefficients between inspiratory EAdi and Q1, Q2, Q3, and Q4 were $0.58 \pm 0.20,0.26 \pm 0.26,0.24 \pm 0.26$, and $0.19 \pm 0.29$, respectively $(p<0.01$; one-way repeated-measures ANOVA).

Individual mean values of EAdi observed during exhalation on prescribed settings of mechanical ventilation are presented in Table 1. Because EAdi stabilized and was compa- rable during Q3 and Q4, we grouped these two quartiles and considered their combined activity as "tonic" activity of the diaphragm. No relationship was found between the level of tonic EAdi and the underlying condition.

The coefficients of variation for EAdi calculated during neural inspiration, Q1, Q2, Q3, and Q4 were 53\%, 44\%, 45\%, $44 \%$, and $71 \%$, respectively.

The mean neural Ti and Te were $0.27( \pm 0.07) \mathrm{s}$ and 0.69 $( \pm 0.24) \mathrm{s}$, respectively, for all 16 patients for the 5-min period at the prescribed PEEP.

Impact of removing PEEP on tonic EAdi $(n=8)$. A subset of the patients ( $n=8$, patients 9-16) were studied for this intervention (Table 1).

The mean and individual levels of tonic EAdi (Q3 and Q4 combined) measured immediately before, during, and immediately after the short period of ZEEP are presented in Figure 4. In all infants but one, tonic activity increased during ZEEP $(p<0.05)$. Returning PEEP to its baseline level, tonic EAdi returned $(p<0.05)$. As indicated in Table 2 , the change from baseline PEEP to ZEEP reduced neural Ti and neural Te, and therefore increased instantaneous neural breathing frequency $(p<0.05)$. Figure 5 demonstrates in one patient the response of the EAdi to removing PEEP.

Reproducibility. The ICCs calculated for the EAdi timing determinations by two investigators were all $>0.75$ and were $0.99,0.83,0.94,0.96$, and 0.93 for neural inspiration, Q1, Q2, Q3, and Q4, respectively.

\section{DISCUSSION}

In the present study, we found in intubated and mechanically ventilated infants that the EAdi after inspiration decreases rapidly during the first half of the neural expiration and plateaus during the second half of the neural expiration to a level that is significantly higher than the noise level. This "tonic" EAdi was observed in every patient and represented $12 \%-15 \%$ of the mean inspiratory EAdi at the prescribed level

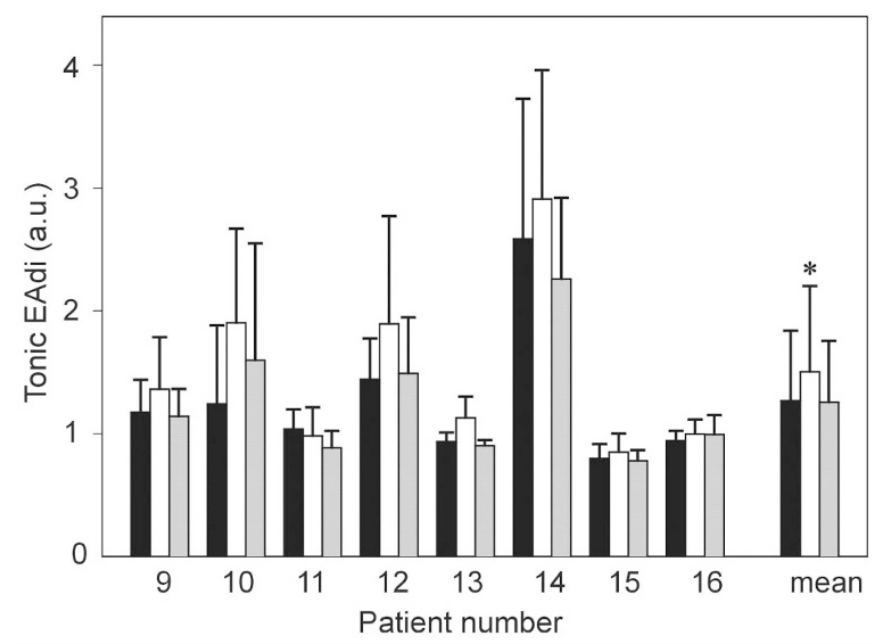

Figure 4. Impact of ZEEP on tonic electrical activity of diaphragm $(n=8)$. Tonic EAdi (combined Q3-Q4) was measured immediately before (solid columns), during (open columns), and after (shaded columns) the ZEEP period. Tonic EAdi significantly increased during the ZEEP period and decreased when PEEP was reapplied $(p<0.01)$. 
Table 2. Effects of ZEEP on neural breathing pattern $(n=8)$

\begin{tabular}{llcll}
\hline & Pre-ZEEP & ZEEP & Post-ZEEP & Significance $(p<0.05)$ \\
\hline Neural Ti (ms) & $347 \pm 65$ & $329 \pm 60$ & $341 \pm 57$ & ZEEP vs Pre-ZEEP \\
Neural Te (ms) & $567 \pm 102$ & $516 \pm 115$ & $619 \pm 149$ & ZEEP vs Post-ZEEP \\
Neural respiratory rate (per min) & $66.6 \pm 8.5$ & $72.6 \pm 11.1$ & $64.4 \pm 11.0$ & ZEEP vs Pre-ZEEPZEEP $v s$ Post-ZEEP \\
Inspiratory EAdi (a.u.) & $3.45 \pm 2.07$ & $4.60 \pm 3.51$ & $3.68 \pm 2.89$ & NS \\
\hline
\end{tabular}

a.u., arbitrary units.

Pre ZEEP

ZEEP

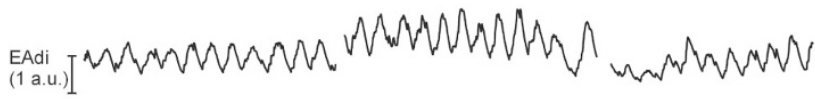

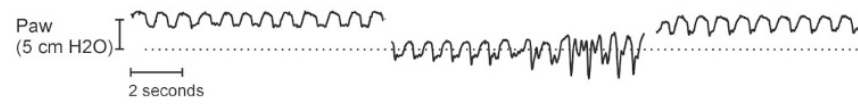

Figure 5. Experimental record during prescribed PEEP, application of ZEEP, and after prescribed PEEP was reapplied. Example in one infant of how removal of the prescribed PEEP resulted in an increase in the tonic activity of the diaphragm.

of PEEP $\left(4 \mathrm{~cm} \mathrm{H}_{2} \mathrm{O}\right)$. Upon removal of PEEP, the tonic EAdi increased and immediately returned (decreased) to its initial level when the prescribed PEEP level was reinstituted. Based on these studies, we could anticipate that there should be a relationship between tonic EAdi and PEEP. Studies in intubated rabbits with lung injury show increased tonic diaphragm activity when no PEEP is applied (unpublished data). Systematic increases in PEEP suppressed the tonic diaphragm activation and increased the phasic activation. The interpretation of these data was that during insufficient PEEP, the diaphragm is activated to defend collapse of lung units, perhaps triggered by receptors in the lung. When the PEEP is increased, it recruits the lung and replaces the action of the tonic diaphragm activation, allowing the diaphragm to perform phasic breathing.

Studies in intubated animals show that continuous negative airway pressure, lung deflation (18), abdominal distention (19), and acute lung injury (unpublished data) result in tonic activation of the diaphragm. The tonic activity was abolished by vagotomy (19; unpublished data). Animal models have also been used to demonstrate that lung deflation $(20,21)$ and pulmonary edema (22) increase the activity of vagal efferents and that direct stimulation of vagal afferents causes tonic activation of the diaphragm (23). Application of PEEP to the intubated airway immediately reduces the activity of the vagal efferents $(20,21)$ and reduces the tonic activity of the diaphragm (unpublished data).

In the clinical setting, infants are frequently provided with CPAP or PEEP to prevent EELV decreases to less than the relaxation volume. Usually this PEEP adjustment is made on an empirical basis. The presence of tonic EAdi in all 16 patients despite applied PEEP levels of 3 to $5 \mathrm{~cm} \mathrm{H}_{2} \mathrm{O}$, which is considered to maintain EELV above relaxation volume in normal infants (24) suggests that the applied PEEP may have been too low or that some tonic activity always occurs in intubated infants. Based on the above, the authors of the present study suggest that the presence of tonic diaphragm activity and its modulation by PEEP may describe a vagal reflex acting to adjust EELV and to prevent a derecruitment of the lungs.

Besides its possible role in the maintenance of EELV, another possible explanation for the presence of tonic EAdi in intubated infants is the presence of nonrespiration-related activity of the diaphragm, e.g. related to postural activities (25) or upper limb movement (26). As well, crying, micturition, and defecation could potentially cause EAdi to be present during expiration. To our knowledge, the importance of this contribution is poorly described in infants; however, there is little support that any of the above reasons should persist over long periods and that it should be observed in all 16 infants (and that ZEEP would increase the tonic EAdi). Kosch and Stark (5) reported that electrical activity measured with surface electrodes positioned on the chest wall was not affected by changes from the supine to upright position. In the present study, infants were studied in the supine position, while not agitated. All periods of crying were excluded from analysis, and only one baby defecated during the study. Therefore, we do not find it likely that factors unrelated to breathing were the cause of our findings of diaphragm activity during the expiratory phase in all infants.

The presence of tonic EAdi may have important physiologic and clinical consequences. The maintained diaphragm activity during expiration likely keeps diaphragm muscle tension higher than if it were relaxed, increasing the diaphragm's metabolism and potentially reducing diaphragm blood circulation (27), both factors that will make the diaphragm more susceptible to peripheral muscular fatigue. In the past, respiratory muscle fatigue was predicted using indices such as the pressure time product or tension time index. Both calculations assume that expiration is passive (28), whereas our findings of EAdi persisting throughout the entire neural expiration may indicate an infinite duty cycle. Diaphragm fatigue could thus be more likely to develop in the case of augmented tonic diaphragm activity. If this is true, this may in part explain the success in treating respiratory failure with CPAP. Monitoring tonic EAdi could then help in the adjustment of ventilator settings to avoid excessive levels.

Another condition that has been associated with tonic EAdi is bronchoconstriction (29). In the present study, there were eight bronchiolitis patients in whom there was no tendency to have increased tonic EAdi relative to the other infants studied. Moreover, diaphragm activation during the expiratory period was significantly present in all patients (with bronchoconstriction or not).

Despite many studies assuming a specific involvement of the diaphragm in controlling EELV in infants $(5,8-10)$, only 
Reis et al. (30) have used esophageal recordings of diaphragm activity and reported that it is uncertain whether the tonic electrical activity measured actually relates to the diaphragm. Esophageal recording of EAdi is the only method currently available to avoid cross-talk interference from muscles other than the diaphragm $(31,32)$. Reis et al. (30), however, reported negligible tonic EAdi from esophageal electrodes, despite the fact that their surface recordings indicated a clear presence of tonic EAdi. These findings are markedly different from the findings of the present study. We can think of at least two possible reasons for the different results obtained by Reis et al. (30) and those obtained in the present study. First, in the present study, a standardized method was implemented to acquire and analyze the diaphragmatic activity (33). The double subtraction technique was used to localize the position of the diaphragm on the electrode array, which eliminates filtering effects related to electrode positioning (13). Reis et al. (30) used a single electrode pair on their esophageal catheter and had no method to verify that the electrodes actually remained in the same position with respect to the diaphragm during both inspiration and expiration. In fact, Beck et al. $(12,34)$ demonstrated that electrode filtering is very powerful and that movement of the diaphragm relative to the electrodes disqualifies all quantitative evaluation of EAdi. Moreover, respiratory movement of the crural diaphragm has been demonstrated to be significant in infants (35). The second potential explanation to explain the discrepancy between the results of Reis et al. and our findings relate to endotracheal intubation. Nonintubated infants exhibit laryngeal braking of expiratory flow that has been associated with the maintenance of EELV and reduced tonic EAdi $(2,7)$. Beck et al. (unpublished data) have recently demonstrated in rabbits with acid-induced lung injury that the tonic activity observed during intubation and ZEEP is reduced after extubation. Thus, because an endotracheal tube was in place for all patients in the present study, upper airway regulation of EELV was not possible, and it may be speculated that tonic EAdi played a more important role in maintaining EELV.

The data obtained in the present study demonstrate the complexity of defining neural inspiration and neural expiration. Our data shows that EAdi persists until the end of expiration. We considered the Q3 and Q4 activity as tonic activity because during this period, the EAdi stopped and the activity remained stable and significantly higher than the noise level. Moreover, the correlation coefficients between inspiration and the different quartiles also show that Q3 and Q4 are not related to inspiration, contrary to Q1. We used the peak activity to delimit the beginning of Q1; however, some diaphragm motor units may not be deactivated, explaining the relationship between Q1 and inspiration. If we believe that the presence of tonic activity is related to the Hering-Breuer deflation sensitive reflex, then it can also explain its presence only during expiration. Moreover, this reflex is involved in a cycle-by-cycle regulation of lung volume could explain the high variability of tonic activity that we measured.

Several problems and potential criticisms inherent to these findings and to the methodology used must be addressed.
Perhaps the most important question that arises from this study is how we can be certain that the signal we measured and quantified during neural expiration was physiologic and originated from the diaphragm and did not simply constitute noise. The cross-correlation algorithm (12) was implemented continuously during inspiration and expiration, every $16 \mathrm{~ms}$, and a predictable pattern of EAdi signals representing diaphragm activity along the array of electrodes (signals were reversed above and below the diaphragm) was observed for both inspiratory EAdi and the Q1-Q4 periods.

The present study showed tonic EAdi in all 16 infants even when PEEP was applied. This could suggest that despite the application of PEEP, certain areas of the lung may not be recruited, and vagal afferents sensitive to lung collapse may be activated. We cannot neglect the fact that the endotracheal tube was suctioned before the study, an action that is known to decrease EELV transiently (36). Therefore, we do not know whether our patients had a normal EELV, even at the baseline PEEP level.

In the present study, we assumed that removing the PEEP level would reduce EELV and that this would alter the tonic EAdi. Although we did not measure the EELV in our patients, several studies report a positive correlation between increasing PEEP and EELV in mechanically ventilated infants $(24,37)$, although the amount of change in EELV per $\mathrm{cm} \mathrm{H}_{2} \mathrm{O}$ of PEEP varies among the studies. Regardless of the magnitude, we believe that it is reasonable to assume that the EELV was reduced by the PEEP removal in the present study.

The validity and reliability of esophageal recordings of EAdi have been criticized for various reasons, including possible contamination by nondiaphragmatic activity, variation in signal strength caused by changes in muscle to electrode distance during respiration, and changes in lung volume (38). However, the methodology that we used for acquisition and analysis of EAdi has been validated to address these problems (33). Contamination of EAdi by nondiaphragmatic activity occurs essentially when surface recording is used (31). Esophageal measurements of EAdi are less subject to contamination because the distance between electrodes and abdominal or intercostal muscles is greater (39). The problems associated with the muscle-to-electrode distance $(12,34)$ and with the influence of cardiac activity and esophageal peristalsis on the signal strength $(13,40)$ have both been resolved. Thus, the method used to analyze and quantify EAdi is reliable and reproducible and accurately provided the data presented in this study.

\section{CONCLUSION}

The findings obtained confirm that the diaphragm remains partially active during expiration of spontaneous breaths in mechanically ventilated infants at PEEP levels that are usually prescribed just before extubation. The results also show that removal of PEEP increases the tonic activity of the diaphragm. This could have potential implications on the management of PEEP in intubated and mechanically ventilated infants. 
Acknowledgments. The authors thank Norman Comtois for technical assistance and Roxanne Trahan for helping to recruit the patients for this study.

\section{REFERENCES}

1. Prechtl HF, van Eykern LA, O'Brien MJ 1977 Respiratory muscle EMG in newborns: a non-intrusive method. Early Hum Dev 1:265-283

2. Lopes J, Muller NL, Bryan MH, Bryan AC 1981 Importance of inspiratory muscle tone in maintenance of FRC in the newborn. J Appl Physiol 51:830-834

3. Stark AR, Cohlan BA, Waggener TB, Frantz ID 3rd, Kosch PC 1987 Regulation of end-expiratory lung volume during sleep in premature infants. J Appl Physiol 62:1117-1123

4. Eichenwald EC, Ungarelli RA, Stark AR 1993 Hypercapnia increases expiratory braking in preterm infants. J Appl Physiol 75:2665-2670

5. Kosch PC, Stark AR 1984 Dynamic maintenance of end-expiratory lung volume in full-term infants. J Appl Physiol 57:1126-1133

6. Colin AA, Wohl ME, Mead J, Ratjen FA, Glass G, Stark AR 1989 Transition from dynamically maintained to relaxed end-expiratory volume in human infants. J Appl Physiol 67:2107-2111

7. Kosch PC, Hutchinson AA, Wozniak JA, Carlo WA, Stark AR 1988 Posterior cricoarytenoid and diaphragm activities during tidal breathing in neonates. J Appl Physiol 64:1968-1978

8. Mortola JP, Milic-Emili J, Noworaj A, Smith B, Fox G, Weeks S 1984 Muscle pressure and flow during expiration in infants. Am Rev Respir Dis 129:49-53

9. Griffiths GB, Noworaj A, Mortola JP 1983 End-expiratory level and breathing pattern in the newborn. J Appl Physiol 55:243-249

10. Elgellab A, Riou Y, Abbazine A, Truffert P, Matran R, Lequien P, Storme L 2001 Effects of nasal continuous positive airway pressure (NCPAP) on breathing pattern in spontaneously breathing premature newborn infants. Intensive Care Med 27:1782-1787

11. Magnenant E, Rakza T, Riou Y, Elgellab A, Matran R, Lequien P, Storme L 2004 Dynamic behavior of respiratory system during nasal continuous positive airway pressure in spontaneously breathing premature newborn infants. Pediatr Pulmonol 37:485-491

12. Beck J, Sinderby C, Lindström L, Grassino A 1996 Influence of bipolar esophageal electrode positioning on measurements of human crural diaphragm electromyogram. J Appl Physiol 81:1434-1449

13. Sinderby CA, Beck JC, Lindström LH, Grassino AE 1997 Enhancement of signal quality in esophageal recordings of diaphragm EMG. J Appl Physiol 82:1370-1377

14. Sinderby C, Beck J, Weinberg J, Spahija J, Grassino A 1998 Voluntary activation of the human diaphragm in health and disease. J Appl Physiol 85:2146-2158

15. Sinderby C, Navalesi P, Beck J, Skrobik Y, Comtois N, Friberg S, Gottfried SB, Lindström L 1999 Neural control of mechanical ventilation in respiratory failure. Nat Med 5:1433-1436

16. Beck J, Tucci M, Emeriaud G, Lacroix J, Sinderby C 2004 Prolonged neural expiratory time induced by mechanical ventilation in infants. Pediatr Res 55:747754

17. Szklo M, Nieto FJ 1999 Epidemiology: Beyond the Basics. Aspen Publishers, Gaithersburg, MD, pp 479-482

18. Meessen NE, van der Grinten CP, Folgering HT, Luijendijk SC 1993 Tonic activity in inspiratory muscles during continuous negative airway pressure. Respir Physiol 92:151-166
19. D'Angelo E, Pecchiari M, Acocella F, Monaco A, Bellemare F 2002 Effects of abdominal distension on breathing pattern and respiratory mechanics in rabbits. Respir Physiol Neurobiol 130:293-304

20. Jonzon A, Pisarri TE, Coleridge JC, Coleridge HM 1986 Rapidly adapting receptor activity in dogs is inversely related to lung compliance. J Appl Physiol 61:19801987

21. Ma A, Bravo M, Kappagoda CT 2003 Responses of bronchial C-fiber afferents of the rabbit to changes in lung compliance. Respir Physiol Neurobiol. 138:155-163

22. Gunawardena S, Ravi K, Longhurst JC, Kaufman MP, Ma A, Bravo M, Kappagoda CT 2002 Responses of C fiber afferents of the rabbit airways and lungs to changes in extra-vascular fluid volume. Respir Physiol Neurobiol 132:239-251

23. Badier M, Jammes Y, Romero-Colomer P, Lemerre C 1989 Tonic activity in inspiratory muscles and phrenic motoneurons by stimulation of vagal afferents. J Appl Physiol 66:1613-1619

24. Thome U, Topfer A, Schaller P, Pohlandt F 1998 The effect of positive end expiratory pressure, peak inspiratory pressure, and inspiratory time on functional residual capacity in mechanically ventilated preterm infants. Eur J Pediatr 157:831837

25. Sinderby C, Ingvarsson P, Sullivan L, Wickstrom I, Lindstrom L 1992 The role of the diaphragm in trunk extension in tetraplegia. Paraplegia 30:389-395

26. Hodges PW, Gandevia SC 2000 Activation of the human diaphragm during a repetitive postural task. J Physiol 522:165-175

27. Hussain SN 1996 Regulation of ventilatory muscle blood flow. J Appl Physio 81:1455-1468

28. Bellemare F, Grassino A 1982 Effect of pressure and timing of contraction on human diaphragm fatigue. J Appl Physiol 53:1190-1195

29. Muller N, Bryan AC, Zamel N 1981 Tonic inspiratory muscle activity as a cause of hyperinflation in asthma. J Appl Physiol 50:279-282

30. Reis FJ, Cates DB, Landriault LV, Rigatto H 1994 Diaphragmatic activity and ventilation in preterm infants. I. The effects of sleep state. Biol Neonate 65:16-24

31. Sinderby C, Friberg S, Comtois N, Grassino A 1996 Chest wall muscle cross talk in canine costal diaphragm electromyogram. J Appl Physiol 81:2312-2327

32. Sharp JT, Hammond MD, Aranda AU, Rocha RD 1993 Comparison of diaphragm EMG centroid frequencies: esophageal versus chest surface leads. Am Rev Respir Dis 147:764-767

33. Aldrich TK, Sinderby C, McKenzie DK, Estenne M, Gandevia SC 2002 ATS/ERS statement on respiratory muscle testing-electrophysiologic techniques for the assessment of respiratory muscle function. Am J Respir Crit Care Med 166:518-624

34. Beck J, Sinderby C, Weinberg J, Grassino A 1995 Effects of muscle-to-electrode distance on the human diaphragm electromyogram. J Appl Physiol 79:975-985

35. Laing IA, Teele RL, Stark AR 1988 Diaphragmatic movement in newborn infants J Pediatr 112:638-643

36. Choong K, Chatrkaw P, Frndova H, Cox PN 2003 Comparison of loss in lung volume with open versus in-line catheter endotracheal suctioning. Pediatr Crit Care Med 4:69-73

37. Schibler A, Henning R 2001 Measurement of functional residual capacity in rabbit and children using an ultrasonic flow meter. Pediatr Res 49:581-588

38. Gandevia SC, McKenzie DK 1986 Human diaphragmatic EMG: changes with lung volume and posture during supramaximal phrenic stimulation. J Appl Physiol 60:1420-1428

39. Petit JM, Milic-Emili G, Delhez L 1960 Examination of the electrical activity of the diaphragm by the esophageal approach in the normal man. J Physiol (Paris) 52:190-191

40. Sinderby C, Lindstrom L, Grassino AE 1995 Automatic assessment of electromyogram quality. J Appl Physiol 79:1803-1815 